\title{
Estaciones de ferrocarril de la Compañía del Norte en España. Intervención y reuso
}

\author{
Aurora María Martínez Corral
}

\section{Objeto de estudio}

Una estación puede definirse como un conjunto de edificios y de espacios destinados a diversas funciones relacionadas, todas ellas, con el ferrocarril: edificio de viajeros, cochera para carruajes y maquinaria, almacenes, muelles cubiertos, espacio de vías, recinto exterior de viajeros y vehículos, talleres, viviendas para empleados, etc. Una característica fundamental que se debe tener en cuenta en las estaciones ferroviarias es la dualidad existente entre el edificio de viajeros -vinculado a la arquitectura civil y pública, con imagen a la ciudad y con carácter representativo- y el espacio destinado a los trenes -vinculado a la ingeniería, libre de estilos y prejuicios y claramente relacionado con la arquitectura industrial y del hierro. Este contraste, resuelto de diferentes maneras con mayor o menor acierto, es otro de los rasgos peculiares que rodean a las estaciones. Por ejemplo, en las estaciones de Atocha y P. Pío esta dualidad está compensada, mientras que en la estación de Delicias predomina el aspecto industrial. En la mayoría de las estaciones de la Compañía del Norte, por la tipología empleada de edificio único alargado, resulta habitual que la integración entre ambos elementos se reduzca a una simple unión lateral. Dentro de las estaciones el resto de edificios e instalaciones que albergan las distintas funciones realizadas tradicionalmente en ellas también son dignos de estudio y del reconocimiento como patrimonio que se debe conservar. No obstante, por acotar, el presente análisis se ciñe al edificio de viajeros y al espacio cubierto de andenes y vías de las principales estaciones de la Compañía del Norte en España.

\footnotetext{
Figura 1:

Estación de Burgos.

Fachada recayente

a andenes $y$ vías

Fuente:

Autora, 2011

Figura 2:

Estación de Delicias,

Madrid. Fachada

acceso principal

Fuente:

Autora, 2010
}
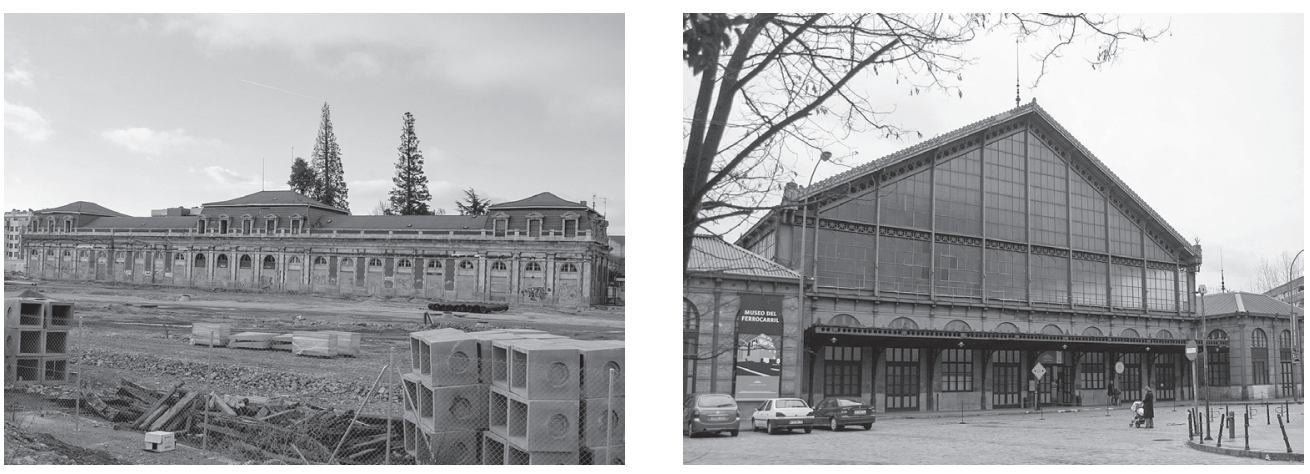

* Cómo citar este artículo: Martínez, A. (2013). Estaciones de ferrocarril de la Compañía del Norte en España. Intervención y reuso. Apuntes, 26(2), 24-37. http://dx.doi.org/10.11144/Javeriana.APC26-2.efcn 


\title{
Estaciones de ferrocarril de la Compañía del Norte en España. Intervención y reuso
}

\author{
Railway Stations from the Compañia del Norte in Spain. Intervention and Reuse \\ Estações ferroviárias da Compañia del Norte na Espanha. Intervenção e reúso
}

\section{Aurora María Martínez Corral}

aumarcor@csa.upv.es

Arquitecta de la Universidad Politécnica de Valencia. Doctora en ingeniería del terreno y en construcciones arquitectónicas. Profesora asociada en el Departamento de Construcciones Arquitectónicas de la Universidad Politécnica de Valencia.

Resumen

¿Intervenir para reutilizar? ¿Intervenir mínimamente para conservar? ¿Usar, reusar, cambiar el uso? Distintos conceptos, cambiantes según sociedades y modas, que conllevan a muy diferentes soluciones, así como también afectan directamente el grado de percepción y valoración social de lo catalogado o reconocido como monumento. Un recorrido por las principales estaciones de la Compañía de los Caminos de Hierro del Norte en España permite observar estas cuestiones y da pie a reflexionar sobre ellas y sus resultados concretos para considerar las conclusiones en futuras intervenciones. El presente trabajo analiza el tipo y grado de intervención existente en la selección realizada a partir del examen de la materialidad constructiva de la intervención, el uso, el entorno, así como el grado de mantenimiento, entre otros factores, y de la reflexión sobre ello. La eficacia de las figuras jurídicas de protección y su aplicación a casos concretos es otra de las cuestiones tratadas. Comparar estaciones poco intervenidas, como Medina del Campo, y algunas casi abandonadas, como la estación de Canfranc, con otras profusamente intervenidas, como la estación de Valencia, para luego valorar el grado de apreciación social de estos monumentos del patrimonio industrial, puede permitir vislumbrar caminos por seguir y ayudar a establecer pautas básicas así como criterios claros de intervención.

Palabras clave: patrimonio industrial; arqueología; monumento histórico; conservación bienes culturales; estación de tren

\section{Abstract}

Intervening to reuse? Intervening minimally to conserve? Does using, reusing, change the use? Different concepts, changing as societies and fashion, leading to very different solutions, and also directly affecting the degree of social perception and valuation of that listed or recognized as monument. A tour of the main stations of the Compañía de los Caminos de Hierro del Norte (Company of the Iron Ways of the North) in Spain allow analyzing these issues and gives rise to reflect on them and their concrete results to consider the findings in future interventions. This paper analyzes the type and degree of intervention in the selection made from the examination of the constructive materiality of the intervention, use, environment, and the degree of maintenance, among other factors, and the reflection on it. The effectiveness of legal protection figures and their application to specific cases is another of the issues. Comparing little intervened stations, as Medina del Campo, and some almost abandoned, as Canfranc station, with other widely tapped as Valencia station and then assess the degree of social appreciation of these monuments of industrial heritage, may allow a glimpse on the ways to follow and help establish basic guidelines as well as clear criteria for intervention.

Keywords: industrial heritage; archeology; historical monument; conservation of cultural property; Train Station

\section{Resumo}

¿Intervir para reutilizar? ¿Intervir minimamente para preservar? ¿Usar, reusar, alterar o uso? Diferentes conceitos, que mudam de acordo às sociedades e às modas, levando para soluções bem diferentes, mesmo como eles estão afetando diretamente o grau de percepção e avaliação social do catalogado ou reconhecido como monumento. Um percurso pelas principais estações da Compañía de los Caminos de Hierro del Norte na Espanha permite observar estas questões e refletir sobre elas e os resultados concretos para considerarem as conclusões em futuras intervenções. 0 presente trabalho analisa o tipo e grau de intervenção existente na seleção feita a partir do exame da materialidade construtiva da intervenção, uso e entorno, bem como o nível de mantimento, entre outros fatores, e

Articulo de Investigación da reflexão sobre isso. A eficácia das figuras jurídicas de proteção e sua aplicação em casos concretos é outro dos assuntos tratados. Comparar estações pouco intervindas, como Medina del Campo, e algumas quase abandonadas, como a estação de Canfranc, com outras profusamente intervindas, como a estação de Valencia, para avaliar após o grau de apreciação social destes monumentos do patrimônio industrial, pode permitir vislumbrar caminhos para seguir e ajudar a estabelecer pautas básicas bem como critérios claros de intervenção.

Palavras-chave: patrimônio industrial; arqueologia; monumento histórico; conservação bens culturais; estação ferroviária

\section{abril de 2013}

Aceptación: $15 \mathrm{de}$

Disponible en línea: 20 de diciembre de 2013 
Las estaciones de la Compañía del Norte se caracterizan, especialmente, por su afrancesamiento y uniformidad estilística, independientemente del lugar de ubicación ${ }^{1}$. Esta compañía tenía una "imagen de marca" que únicamente superó $^{2}$ el arquitecto Demetrio Ribes. La estandarización y copia de proyectos en diferentes localidades era una práctica habitual en la construcción de las estaciones, independientemente del entorno y de la imagen arquitectónica del lugar. Como rasgo positivo se destaca que la estandarización presupone un estudio en profundidad, perfeccionado, de distribución y composición de un edificio. Respecto al objeto del presente estudio, dicha característica permite observar con mayor claridad, dado que el punto de partida es similar, la evolución experimentada en los diferentes edificios analizados y el estado actual de los mismos.

\section{Concepto de patrimonio cultural aplicado a las estaciones. Estado actual}

En el presente artículo no se entra a discernir acerca del significado de patrimonio cultural, así como tampoco respecto de los cambios que este ha experimentado a lo largo de la historia, o por qué las estaciones forman parte del mismo, ya que ello ha sido ampliamente tratado por distintos investigadores ${ }^{3}$. Actualmente es evidente que las estaciones de ferrocarril son patrimonio, aunque tienen escasa historia y están todavía poco valoradas ${ }^{4}$; concretamente se trataría de conjuntos arquitectónicos analizados en su globalidad, ya que ostentan valores de uso, formales y simbólicos-significativos, son modelos de referencia y, en definitiva, también son un conjunto de identidad colectiva (Llull, 2005). El hecho de que estén “vivos" por mantener el uso original -aunque, como expone 1. Aguilar ${ }^{5}$, uso, funcionalidad y utilidad pueden incidir en detrimento de su revalorización como monumento-, supone un valor adicional ya que les permite evolucionar conjuntamente con la sociedad y analizar esta evolución mediante la observación de los cambios que produce. Esta relación uso-revalorización monumental incide en los casos estudiados. Sin embargo, estas evidencias se ven en el papel pero no en la consideración social. Ni siquiera los técnicos encargados de la intervención son conscientes del valor patrimonial de las estaciones y ello se percibe en los resultados de su trabajo.
Por otra parte, en las intervenciones practicadas por técnicos conscientes de este valor tampoco se debe permitir ni la más mínima manifestación de ego, pues el arquitecto que redacta un proyecto de ejecución debe partir de un estudio exhaustivo previo -elaborado por un equipo multidisciplinar- de forma que pueda interpretar el monumento para obtener, a partir de esta, la solución, sin poner nada propio más que la técnica adecuada. El arquitecto restaurador no es más que un eslabón en la cadena que es la historia del monumento (Álvarez: 2008). A este respecto se citan las palabras de A. Siza:

Respeto, humildad a la historia. Comprensión del carácter de los sitios. Preservación de la integridad de la obra arquitectónica que debe valorarse tal cual es. En ningún caso deben prevalecer cuestiones de gusto. Todos los edificios antiguos tienen mil oportunidades de intervención en continuidad.

Hay que descubrir el sentido de las cosas sin menospreciar (Siza, 2011).

Algo parecido acontece con la arquitectura del movimiento moderno y con el patrimonio industrial en su conjunto ${ }^{6}$. Factores como la escasa antigüedad, la insuficiente difusión y la “democratización” de este patrimonio ${ }^{7}$, la falta de revalorización pública por parte de las autoridades (en el caso de las estaciones pesa más en la conciencia social las connotaciones negativas asociadas al ferrocarril; efecto barrera, degradación, contaminación acústica, etc., que las positivas: monumentales, valores tecnológicos, valores emocionales, etc.), y entre otros añadiría el modo de vida actual en el que la prisa impide la contemplación. Todo lo anterior conlleva a las numerosas demoliciones acontecidas, a las intervenciones exentas de delicadeza o al absoluto abandono, aunque, en algunas ocasiones, quizá sea la mejor de las opciones.

Es obvio y ampliamente manifestado que si se quiere preservar el patrimonio es necesario implicar a todos los sectores sociales y sensibilizar adecuadamente a la población. En los siguientes apartados se muestran diversas intervenciones inadecuadas en mayor o menor grado que han alienado el sentido original de la obra, lo cual, grave de por sí-por la pérdida de contexto, de espacialidad, de materialidad original, de uso, etc.-, lo es mucho más debido a que va transformando
1 Las fachadas realizadas por los arquitectos Percier (17641838) y Fontaine (1762-1853), creadores de los estilos directorio e imperio, son un claro ejemplo de este estilo y han servido de inspiración a numerosas estaciones, especialmente de la Compañía del Norte. Concretamente, para el caso español, las primeras estaciones fueron mayoritariamente obra de ingenieros ingleses, o franceses, que, simplemente, adaptaron

los proyectos ya ejecutados para otras estaciones. Entre los años 1870 y 1900 la

cosa cambia y se produce la expansión de las compañías; este periodo de construcción es el de mayor número de estaciones en nuestro país, y es cuando aparece la necesidad de dar más ornato y representatividad a los edificios de viajeros. En este segundo periodo se observa una desaparición del influjo inglés y, en cambio, se observa una creciente y mayor presencia francesa. Es el tiempo de las estaciones monumentales, que coincide con auge de las grandes compañías. También se produce en esta etapa el mayor número de construcción de estaciones con carácter definitivo en nuestro estado. El tercer periodo que puede establecerse en relación con la construcción de estaciones en nuestro país, se sitúa entre 1902 y 1941 (creación de la Red Nacional de Ferrocarriles Españoles, renfe). Es significativo de esta etapa el hecho de que desaparece por completo la dependencia del ingeniero extranjero. Las estaciones quedan, al fin, en manos de profesionales del país.

2 Ello se observa claramente en las estaciones de Valencia principalmente y en la ampliación de la estación de Barcelona Nord. Su sello también se muestra en las estaciones de Vic, Palencia y León, aunque más discretamente.

3 Concretamente respecto de las estaciones resulta imprescindible estudiar el fecundo trabajo de I. Aguilar. También son relevantes los estudios de Mercedes López, entre otros.

4 Esto ocurre generalmente con el patrimonio industrial y también con el patrimonio de la obra pública y del movimiento moderno. El alejamiento de cánones tradicionales, el empleo de nuevos materiales, la funcionalidad y los usos, y la falta de antigüedad total pueden ser los factores causantes principales.

5 La profesora Aguilar 
expone esta idea en numerosas publicaciones.

Véase la bibliografía.

6 Respecto al movimiento moderno, consultar: las conclusiones de la Conferencia Internacional sobre los

Criterios de Intervención en el Patrimonio Arquitectónico del Siglo XX (CAH 20th), Madrid, junio de 2011.

Respecto al patrimonio industrial consultar: Plan Nacional de Patrimonio Industrial, PNPI, 2011. "Desaparición acelerada del patrimonio industrial".

7 Debe tenerse en cuenta que, aproximadamente en los tres últimos siglos, la manera de ver la cultura en Europa ha pasado de ser algo puramente elitista a una preocupación política de primer nivel, aunque con un defecto importante: categorizar los monumentos casi exclusivamente por su antigüedad cronológica. Esto deja prácticamente fuera tanto a las estaciones de ferrocarril como a las obras del movimiento moderno. Afortunadamente, desde principios del XX (Riegl, 1903) se han ido añadiendo valores de contemporaneidad a la vez que crecía la tolerancia hacía manifestaciones culturales y estilos alejados de los cánones tradicionales.

8 Hay actividades como trenes turísticos o a vapor en algunas localidades, pero no están encaminados a la pues ta en valor de las estaciones.

9 La situación se agrava para el caso de las estaciones en particular y del patrimonio industrial en general, ya que sobre ellos existe poca conciencia social y la actitud destructiva se hace patente en muchos casos. Se concretará esta cuestión al trata los casos particulares de las estaciones intervenidas.

10 La propia Ley General de Patrimonio Histórico, vigente en España desde 1985, tiene muy en cuenta este factor al confirmar el valo relativo del patrimonio y su ineludible significación social. El patrimonio es entendido así como "riqueza colectiva" y el objetivo de su regulación es la protección, acrecentamiento y transmisión de la misma a las generaciones futuras.

11 Información extraída de Registro de Bienes de Interés Cultural, RBIC (Registro de Bienes de Interés Cultural), del MECD (Ministerio de Educación, Cultura y Deporte de España). el concepto original -en este caso ser "edificio de viajeros de una estación”- en la mente colectiva, hasta llegar a una pérdida total de referencias y, por tanto, del sentido que induce a la conservación de una determinada obra.

La estrategia política respecto al patrimonio industrial en general es "democratizar la cultura" estableciendo que se asocie el tiempo de ocio con el disfrute cultural (viajes culturales, exposiciones, muestras, ecomuseos, museos temáticos etc.), pero las estaciones actualmente quedan fuera de este circuito ${ }^{8}$. Además, esta táctica supone un conocimiento general muy superficial y banal de los monumentos, y en algunos casos se añade una actitud agresiva hacia estos ${ }^{9}$ por parte de algunos turistas (Llull, 2005) lo que conlleva a pensar que esta estrategia no conduce finalmente a la valorización del patrimonio.

En cualquier caso, la creciente descentralización de competencias obliga a que cada vez sea la ciudadanía más cercana al monumento la que deba protegerlo. Lamentablemente, políticos y expertos ven con recelo la participación ciudadana y cuentan poco con sus opiniones, aunque queda constatado que la conducta de la sociedad civil es de enorme trascendencia respecto de la conservación y de mayor impacto que el papel desempeñado por las instituciones gubernamentales ${ }^{10}$, aunque, por otro lado, la ciudadanía, tal vez por conformismo y apatía, no ha tenido reparos en delegar en los poderes públicos la entera responsabilidad de conservar, restaurar, difundir y gestionar los bienes culturales (Llull, 2005); y estos, en las respectivas legislaciones autonómicas (excepto la Ley Balear 12/98; la Ley Madrileña 10/98, la Ley Cántabra 11/98 y sobre todo, la Ley Asturiana 1/2001 y sus modificaciones (8/2010 y 1/2011) (Pardo, 2010), tampoco están a la altura de esa declaración de "riqueza colectiva" y con la función social que supone el patrimonio según la Ley General de Patrimonio Histórico. La coyuntura actual dificulta todavía más esta cuestión puesto que al conformismo y apatía de la ciudadanía quizá habría que añadir la existencia de graves problemas fundamentales que obligatoriamente postergan las soluciones de aquellos relacionados con el patrimonio.

\section{Protección jurídica de las estaciones de Norte en España. Estado de la cuestión}

La declaración de bien de interés cultural supone la máxima figura de protección jurídica existente en nuestro país. A pesar de distintos matices en las diversas legislaciones autonómicas, conlleva una serie de medidas generales y otras específicas encaminadas a evitar alteraciones (incluso menores) de los elementos así declarados.

En la actualidad once estaciones de ferrocarril están incluidas en el Registro General de Bienes de Interés Cultural ${ }^{11}$, ello supone aproximadamente un 10\% del total de Bienes Industriales Inmuebles inscritos ${ }^{12}$. En la siguiente tabla se detallan los mismos:

Del análisis de los datos contenidos en la tabla adjunta se hacen las siguientes deducciones:

Prácticamente en la mitad de las estaciones incluidas únicamente se ha incoado el correspondiente expediente a pesar de que, como ocurre en las estaciones de Atocha y Príncipe Pío, hayan transcurrido más de treinta años ${ }^{13}$. Habría que

\section{LISTA DE ESTACIONES CONTENIDAS EN EL RGBIC}

\begin{tabular}{llcr}
\hline \multicolumn{1}{c}{ ESTACIÓN } & AÑO & ESTADO & TIPO \\
\hline E. de Atocha & 1977 & Incoado & Monumento \\
E. de P. Pío & 1977 & Incoado & Monumento \\
E. de Zaragoza & 1980 & Incoado & Monumento \\
E. de Segovia & 1982 & Incoado & Monumento \\
E. Norte de Valencia & 1983 & Declarado & Monumento \\
E. de Almería & 1985 & Declarado & Monumento \\
E. Plaza de Armas & 1990 & Declarado & Monumento \\
Antigua E. de Vigo & 1990 & Incoado (A. Preventiva) & Monumento \\
E. de Toledo & 1991 & Declarado & Monumento \\
Estaciones FFCC Sóller a Inca & 1996 & Incoado (A. Preventiva) & Conjunto histórico \\
E. de Canfranc & 2002 & Declarado & Monumento \\
\hline
\end{tabular}

Tabla. 1. Estaciones BIC (incoadas o declaradas) españolas. Orden cronológico. 


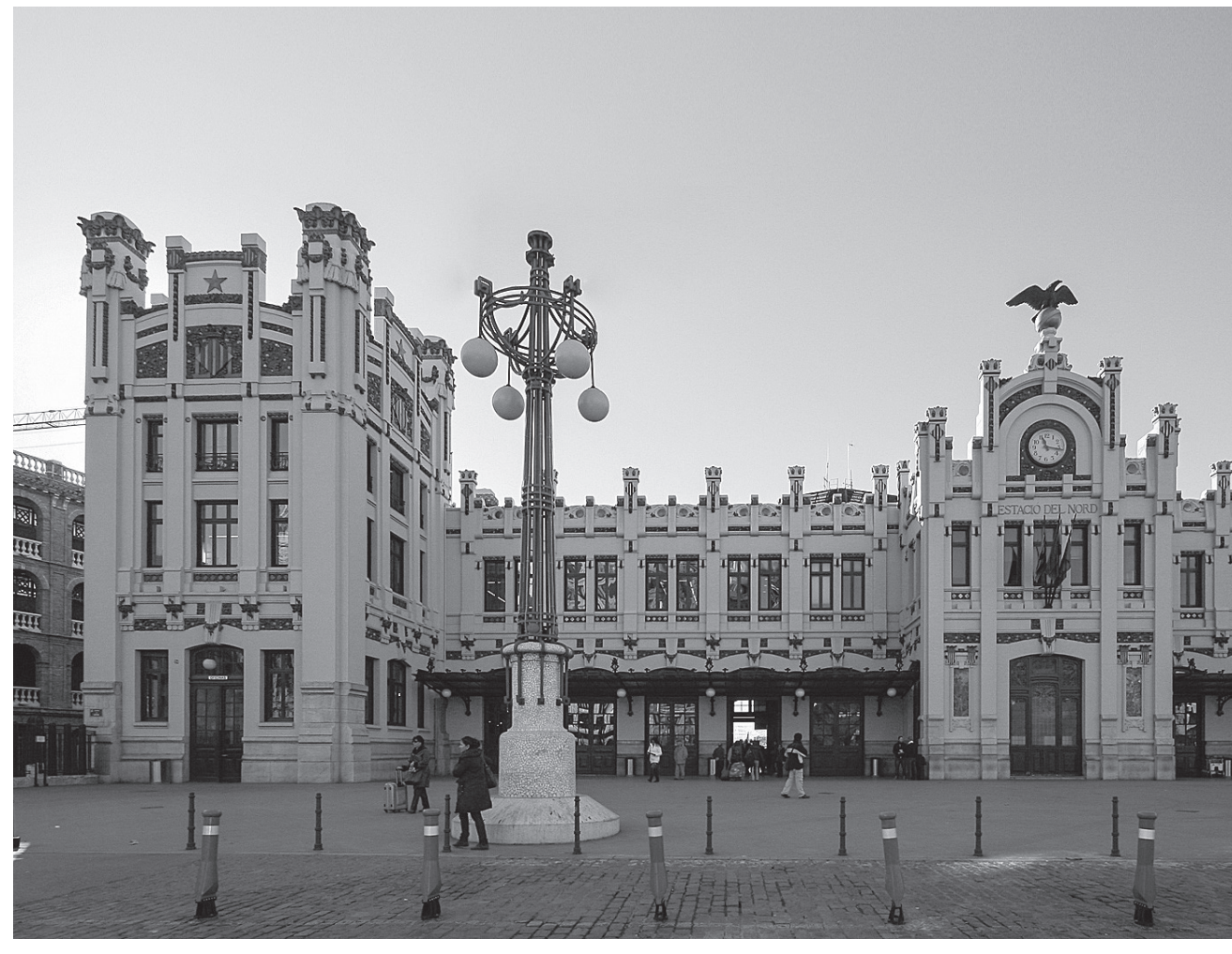

preguntarse si, legalmente, la protección jurídica continúa activa así como las posibles consecuencias que puede provocar esta situación de expedientes inconclusos.

En todos los casos, excepto en las estaciones de Sóller a Inca, tanto las incoaciones como las declaraciones se hacen en la categoría de monumento y la protección parece restringirse al edificio de viajeros exclusivamente. En el caso de las estaciones debería hablarse de conjuntos históricos puesto que poseen un interés claro (valores históricos, tecnológicos, artísticos, sociales, etc.), sus edificios e instalaciones forman un conjunto coherente y están agrupados de forma que constituyen unidades geográficas delimitadas (Aguilar, 2006). pero, independientemente de esta u otra definición, debe quedar claramente expuesto que el objeto de protección es la totalidad del conjunto de edificios e instalaciones que comprende la estación ya que, como se detallará en alguno de los casos estudiados, esta protección no se ha extendido a todo el conjunto de la estación.

La primera de las estaciones declarada monumento fue la estación del Norte de Valencia. En el presente artículo se analiza si dicha protección ha resultado efectiva mediante el análisis de las diversas intervenciones -mayores y menoresrealizadas en el edificio de viajeros. Indicar que,
Figura 3:

Estación de Valencia,

Fachada recayente

a andenes $y$ vías

Fuente:

Autora, 2011

(2010) indica que, en 2008, eran 100 los bienes industriales inscritos en el RBIC.

13 Aunque las diferentes respecto del conjunto de la estación, el proyecto "Parque Central"14 prevé la conservación de algunos de los almacenes, actualmente muy alterados o deteriorados. Es decir, el mantenimiento y consolidación ha sido prácticamente nulo, y en los casos donde se ha llevado a cabo, profundamente alienante. No se aprecia sensibilidad hacia los mismos cuando se contempla su traslado y reconstrucción, ya que ello, unido al cambio de uso, descontextualiza y desvirtúa completamente el edificio.

A este respecto también la antigua estación de Vigo, incoada pero con anotación preventiva, ha sido reducida a un montón de piedras numeradas y acopiadas. ¿Qué tiene esto que ver con el concepto actual de monumento? ¿Dónde está la preservación de valores culturales, nostálgicos e intangibles?

También los datos del registro resultan imprecisos en algunos casos: por ejemplo, no se define con exactitud la estación de Zaragoza ${ }^{15}$, y tampoco aparece la fecha de declaración ni del boletín correspondiente de la estación de Almería ${ }^{16}$.

Es necesario destacar que no se trata de un registro exhaustivo ya que no se incluyen edificios tan relevantes como la estación de Delicias, incoada como monumento en $1981^{17}$, ni las estaciones de Francia, Zamora, Concordia, Aranjuez legislaciones autonómicas

establecen plazos ligeramente distintos en la tramitación del expediente desde la incoación a la declaración, en los bienes inmuebles este plazo no supera los 20 meses.

14 Ver Plan de Reforma Interior ámbito A.4.1. "Actuación urbanística informativa y justificativa.

15 Se presupone esta estación porque es la única catalogada de la ciudad. El 22 de noviembre de 2002 se publicó en el Boletín Oficial de Aragón la Orden de 24 de octubre de 2002 del Departamento de Cultura y Turismo por la que se declara Bien Catalogado del Patrimonio Cultural Aragonés la Antigua Estación de Ferrocarril Zaragoza-Delicias o de Caminreal, Zaragoza (Fuente: Registro Patrimonio Cultural de Aragón).

16 Sin embargo, esta sí cuenta con número de registro (R.I. 51-0011414-0000).

17 Resolución de 21 de enero de 1981, de la Dirección General de Bellas Artes, Archivos y Bibliotecas, por la cual se acuerda tener por incoado expediente de declaración de monumento histórico-artístico a favor de la estación de ferrocarril de "Las Delicias", de Madrid. BOE No. 42, 18 de febrero de 1981, p. 3731. 'Parque Central'”. Memoria 
o Valladolid, entre otras. Tampoco aparece la estación de El Grao, en Valencia, la más antigua de España que queda en pie, y ni siquiera figura en el registro autonómico de la Comunidad Valenciana como bien inventariado o bien de relevancia local ${ }^{18}$. En cuanto a los registros autonómicos, se debe añadir la falta de consenso respecto de la denominación y catalogación de las estaciones: en unos casos se consideran obra civil, en otros edificios dotacionales del transporte, arquitectura del hierro, etc. ${ }^{19}$

Por último, respecto al ámbito del presente estudio, se identifica que de las estaciones principales de la Compañía del Norte en España solo cuatro de ellas aparecen en el registro analizado. Estaciones como Burgos, Valladolid, Medina del Campo, etc., están inscritas, en el mejor de los casos, en los inventarios autonómicos, circunstancia que, obviamente, va en detrimento de la conservación de este patrimonio.

Analizaremos las diferencias de tratamiento existentes entre unas y otras lo que nos permitirá concluir acerca de la efectividad de la incoación y/o declaración de bien de interés cultural.

\section{Objetivos}

El trabajo realizado muestra las diferentes intervenciones realizadas en los edificios de viajeros de las principales estaciones de la Compañía del Norte en España. Se analizan estas intervenciones pretendiendo demostrar hasta qué punto resulta efectiva la declaración de BIC en la práctica, la importancia del conocimiento exhaustivo previo del elemento que se va a intervenir, las consecuencias del cambio de uso, de espacialidad y la descontextualización, etc., con la finalidad de exponer un estado extrapolable de la cuestión actual respecto de la conservación de las estaciones de ferrocarril en España.

\section{Materiales y métodos}

El presente análisis se basa, en un primer lugar, en el estudio de la historia de la Compañía del Norte en España, de forma que el mismo propicia la selección de sus principales estaciones. Concretamente, el conjunto inicial lo forman 27 estaciones: A Coruña, Lugo, Gijón, Oviedo, León, Valladolid,

Tabla 2. Principales estaciones existentes Ca Norte en España. Datos descriptivos 1.

\begin{tabular}{|c|c|c|c|}
\hline NOMBRE & PROTECCIÓN & ED. ORIGINAL & FECHA CT ED. ORIGINAL \\
\hline Gijón & No & Existente. Ampliada. Reconocible & 1871 \\
\hline Lugo & No & $\begin{array}{l}\text { Existente. Proyecto reconversión } \\
\text { E. Intermodal }\end{array}$ & $1871-1873$ \\
\hline León & No & Existente. Intervenida. Reconocible & 1880 \\
\hline Valladolid & No & Existente. Intervenida. Reconocible & 1890 \\
\hline $\begin{array}{l}\text { Medina } \\
\text { del Campo }\end{array}$ & No & Existente. Poco intervenida. Reconocible & 1896 \\
\hline Segovia & $\begin{array}{l}\text { Sí } \\
\text { BIC incoado }\end{array}$ & $\begin{array}{l}\text { Existente. Intervenida. Reconocible } \\
\text { exteriormente: fachadas y marquesinas }\end{array}$ & 1888 \\
\hline Madrid (P. Pío) & $\begin{array}{l}\text { Sí } \\
\text { BIC incoado }\end{array}$ & $\begin{array}{l}\text { Existente. Muy intervenida. Parcialmente } \\
\text { reconocible: fachadas y cubierta metálica }\end{array}$ & $\begin{array}{l}\text { 1878-1882 } \\
\text { Ampl. } 1928\end{array}$ \\
\hline Palencia & No & $\begin{array}{l}\text { Existente. Intervenida. Reconocible: } \\
\text { fachadas, excepto acceso. Soportes } \\
\text { y espacio marquesinas }\end{array}$ & $1875-1885$ \\
\hline Burgos & No & $\begin{array}{l}\text { Existente. Estado abandono. Reconocibles } \\
\text { fachadas. Pdte. Intervención. En proceso } \\
\text { de adjudicación de redacción de proyecto }\end{array}$ & 1901 \\
\hline Miranda de Ebro & No & Existente. Intervenida. Reconocible & 1862 \\
\hline S. Sebastián & No & Existente. Intervenida. Reconocible & 1880 \\
\hline Canfranc & $\begin{array}{l}\text { Sí } \\
\text { BIC declarado }\end{array}$ & $\begin{array}{l}\text { Existente. Consolidada cub. y st. En } \\
\text { estudio de varios proyectos de intervención }\end{array}$ & $\begin{array}{l}\text { 1913-1918. } \\
\text { Inaug: } 1928 .\end{array}$ \\
\hline Zgz-Arrabal & No & $\begin{array}{l}\text { Existente. Intervenida. } \\
\text { Reconocibles fachadas }\end{array}$ & 1861 \\
\hline Ben-Nord & $\begin{array}{l}\text { Sí } \\
\text { Inventario }\end{array}$ & $\begin{array}{l}\text { Existente. Muy intervenida. Reconocibles } \\
\text { fachadas, ampliación original y cubierta }\end{array}$ & $\begin{array}{l}1^{\text {a }} \text { etapa: } 1859-1865 \\
\text { Ampl.: } 1906-1910\end{array}$ \\
\hline Valencia & $\begin{array}{l}\text { Sí } \\
\text { BIC declarado }\end{array}$ & $\begin{array}{l}\text { Existente. Muy intervenida. } \\
\text { Reconocibles fachadas, parte de } \\
\text { espacios interiores y cubierta }\end{array}$ & 1906 \\
\hline
\end{tabular}

18 Inventario General de Patrimonio Cultural Valenciano

19 También la denominación de BIC cambia en Catalunya y

P. Vasco tomando el nombre de "Bé Cultural D’Interés Nacional" (BCIN) o "Bien Calificado" (BC), respectivamente 
Medina del Campo, Ávila, Segovia, Madrid (Príncipe Pío), Palencia, Santander, Burgos, Miranda de Ebro, Bilbao (Abando), Vitoria, S. Sebastián, Irún, Logroño, Pamplona, Huesca, Canfranc, ZaragozaArrabal, Lleida, Barcelona (Nord), Tarragona y Valencia. Seguidamente se realiza un análisis documental para conocer el estado inicial del edificio, y se procede a una elaboración de fichas para cada una de estas estaciones, las cuales incluyen: datos generales, ubicación, estilo arquitectónico, rasgos compositivos relevantes, tipología, materiales y detalles constructivos característicos. La totalidad de estas fichas conforma uno de los capítulos de mi tesis doctoral.

A continuación se procede a identificar el estado actual de estas estaciones y se observa que han desaparecido los edificios de viajeros originales (o aquellos sustituidos en las etapas de mejoras de la Compañía) ${ }^{20}$ en las estaciones de: A Coruña, Oviedo, Ávila, Santander, Bilbao-Abando,

Tabla 3. Principales estaciones existentes Ca Norte en España. Datos descriptivos 11.

\begin{tabular}{|c|c|c|c|}
\hline ESTACIÓN & USO ACTUAL & INTERVENCIONES MAYORES & INTERVENCIONES MENORES \\
\hline Gijón & $\begin{array}{l}\text { Ferroviario } \\
\text { Museo ffcc }\end{array}$ & $\begin{array}{l}\text { Sí } \\
\text { Nave anexa }\end{array}$ & Carpintería \\
\hline Lugo & Ferroviario & $\begin{array}{l}\text { Sí } \\
\text { Marquesinas acceso y andenes }\end{array}$ & $\begin{array}{l}\text { Chimeneas. Señalización. } \\
\text { Color fachada (?) }\end{array}$ \\
\hline León & Sin uso & $\begin{array}{l}\text { Sí } \\
\text { Acceso (marquesina y mod. } \\
\text { huecos) Anexo cuerpo f. trasera }\end{array}$ & $\begin{array}{l}\text { Carpintería. Señalización. } \\
\text { Chapa cubierta andenes }\end{array}$ \\
\hline Valladolid & Ferroviario & $\begin{array}{l}\text { No } \\
\text { (Pasarela ave) }\end{array}$ & $\begin{array}{l}\text { Carpintería. Señalización. } \\
\text { Chapa cubierta andenes. } \\
\text { Acabado fachada }\end{array}$ \\
\hline Medina del Campo & Ferroviario & No & $\begin{array}{l}\text { Carpintería. Señalización. } \\
\text { Chapa cubierta andenes }\end{array}$ \\
\hline Segovia & Ferroviario & No & $\begin{array}{l}\text { Carpintería. Señalización. } \\
\text { Chapa cubierta andenes }\end{array}$ \\
\hline Madrid (P. Pío) & $\begin{array}{l}\text { Comercial- } \\
\text { E. intermodal }\end{array}$ & $\begin{array}{l}\text { Sí } \\
\text { Anexión cuerpos. Galería } \\
\text { comercial. Profunda distorsión } \\
\text { interior. Perforación metro }\end{array}$ & $\begin{array}{l}\text { Acabado cubierta ed. viajeros. } \\
\text { Instalaciones. Carpintería. Color } \\
\text { fachada. Señalización. Color } \\
\text { fachada (?) [o acabado (?)] }\end{array}$ \\
\hline Palencia & Ferroviario & $\begin{array}{l}\text { Sí } \\
\text { Acceso }\end{array}$ & $\begin{array}{l}\text { Carpintería. Señalización. } \\
\text { Chapa cubierta andenes }\end{array}$ \\
\hline Burgos & Sin uso & $\begin{array}{l}\text { Sí } \\
\text { Eliminación marquesina } \\
\text { andenes }\end{array}$ & $\begin{array}{l}\text { Abandono. Por intervenir. } \\
\text { En proceso }\end{array}$ \\
\hline Miranda de Ebro & Ferroviario & No & Intervenida recientemente \\
\hline S. Sebastián & Ferroviario & No & $\begin{array}{l}\text { Señalización. Carpintería (al } \\
\text { menos acceso). Acabado } \\
\text { cubierta metálica andenes } \\
\text { y marquesina acceso }\end{array}$ \\
\hline Canfranc & $\begin{array}{l}\text { Sin uso } \\
\text { (solo apeadero } \\
\text { uso ferroviario) }\end{array}$ & $\begin{array}{l}\text { Sí } \\
\text { Cúpulas, cubierta. Pináculos } \\
\text { fuera de escala y chimenea }\end{array}$ & $\begin{array}{l}\text { Abandono } \\
\text { Por intervenir }\end{array}$ \\
\hline Zaragoza-Arrabal & Centro cívico & $\begin{array}{l}\mathrm{Si} \\
\text { Eliminación marquesina } \\
\text { andenes }\end{array}$ & $\begin{array}{l}\text { Carpintería. Color } \\
\text { de fachada (?) }\end{array}$ \\
\hline Barcelona-Nord & $\begin{array}{l}\text { Polideportivo- } \\
\text { Comisaria-E. } \\
\text { autobuses }\end{array}$ & $\begin{array}{l}\text { Sí } \\
\text { Marquesina acceso. } \\
\text { Distorsión interior: pistas } \\
\text { deportivas, estancias } \\
\text { comisaría y e. de autobuses } \\
\text { Pasarelas y acceso } \\
\text { lateral e. autobuses }\end{array}$ & $\begin{array}{l}\text { Carpintería. Color fachada } \\
\text { Cubierta metálica andenes } \\
\text { Modificación mat. interior }\end{array}$ \\
\hline Valencia & Ferroviario & $\begin{array}{l}\text { Sí } \\
\text { Distorsión interior: galería } \\
\text { comercial. Marquesina } \\
\text { f. lateral derecha }\end{array}$ & $\begin{array}{l}\text { Carpinterías. Señalización. } \\
\text { Mat. acabado cubierta bóveda. } \\
\text { Color fachada. Sustitución } \\
\text { vidrios. Zócalo pétreo. } \\
\text { Modificación mat. interior }\end{array}$ \\
\hline
\end{tabular}

Fuente: MECD - FFE

Elaboración propia tras visitas in situ (2007-2012)

20 La Compañía del Norte en España se desarrolló en cuatro etapas principalmente: $1^{\text {a }}$ etapa: $1859-1874$

$2^{\text {a }}$ etapa: $1874-1900$

- $3^{\text {a }}$ etapa: $1900-1913$

$4^{\mathrm{a}}$ etapa: 1913-1936.

Fue a partir de 1870, es decir, a comienzos de la $2^{a}$ etapa, cuando la compañía comenzó un plan de reforma y construcción para el mejoramiento de sus líneas y que se prolongó durante toda la $3^{\mathrm{a}}$ etapa. Este periodo se inicia con el anteproyecto de la estación de Príncipe Pío en Madrid (1873), después llegaron los de Santander (1876), San Sebastián (1880), Irún (1889), Valladolid (1890-1891), Medina del Campo (1896), Burgos (1901), Valencia Norte (1906-1918) y BarcelonaVilanova (1914-1918) 


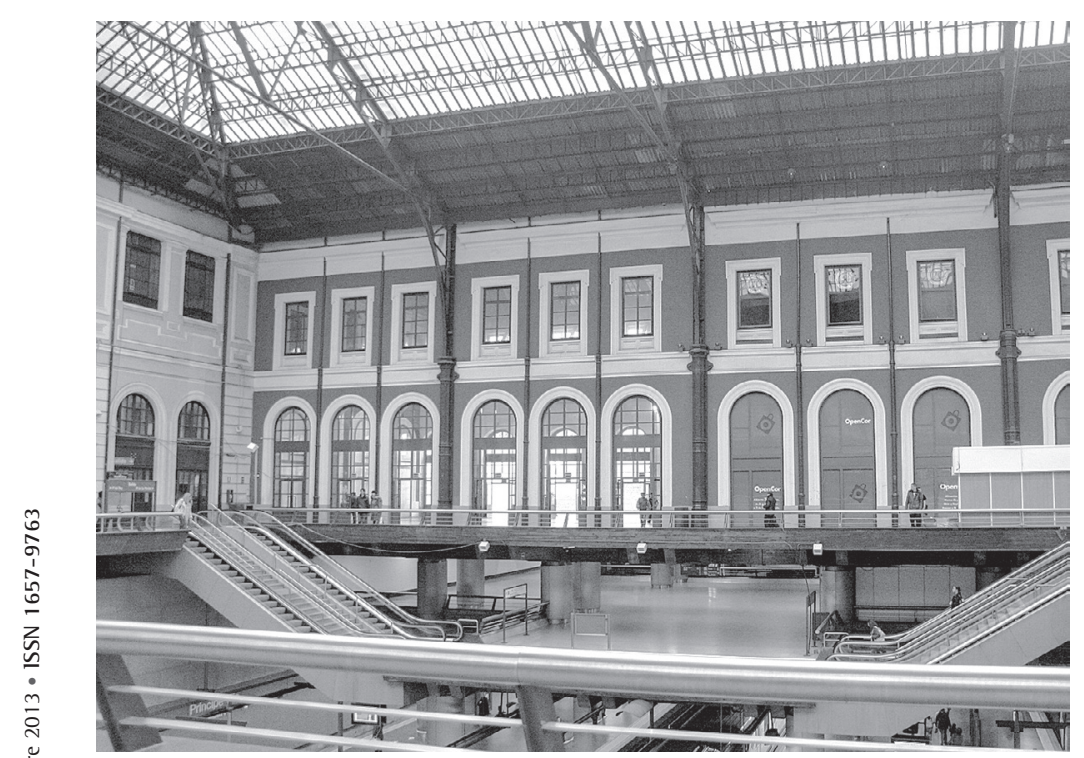

Figura 4: Vitoria, Irún, Logroño, Pamplona, Huesca, Lleida Estación Príncipe Pío, y Tarragona. Así, del total han desaparecido 12 Madrid. Vaciado bajo
el edificio de viajeros

Fuente: Autora, 2010

21 Ya la ampliación de los años veinte (1928) muestra una insensibilidad total hacia lo preexistente y hacia el uso de los nuevos materiales al ocultar la estructura metálica con cúpulas y órdenes clásicos en hormigón armado

22 El estudio y datación de las diversas intervenciones sufridas en la estación de Valencia están detalladas en el capítulo correspondiente de mi tesis doctoral aprecia poca sensibilidad hacia el edificio preexistente ${ }^{21}$ : la estación se ha convertido en un centro comercial y en un intercambiador modal. Se han producido drásticos cambios de espacialidad interior (los andenes interiores han sido sustituido por tiendas); igualmente de materialidad, pues ni siquiera se ha respetado el característico ladrillo rojo aplantillado de Norte, y de uso. Básicamente, el interior del edificio de viajeros ha sido vaciado dejando solo las fachadas, cual máscaras inertes, desprovistas de sus recuerdos, y fuera de lugar. El vestíbulo lo ocupa ahora la estructura metálica tubular de arriostramiento de estas fachadas. La obra ingenieril ha sido de gran complejidad (Menéndez et al., 1996), sin embargo, se debe resaltar que se han profanado hasta los cimientos ya que las vías pasan por debajo del edificio de viajeros, siendo sorprendente la imagen que se percibe al visitarlo. La cuestión reside en si este tipo de actuaciones son para conservar o para reutilizar un determinado edificio a modo de contenedor, ya que esta descontextualización y alienación provoca en la conciencia colectiva un cambio de significado a lo largo del tiempo.

3. La estación de Barcelona Nord fue reconvertida en 1992 en sede olímpica. Actualmente, tanto la parte ampliada (D. Ribes 1909-1910) como el espacio de andenes y vías son un polideportivo municipal. En el lugar que ocupaban los andenes ahora hay unas canchas de baloncesto, al lado de las carpinterías diseñadas por Ribes, y junto a la gran fachada de vidrio y acero, hay mesas de ping-pong. El edificio original (P. de Andrés i Puigdollers 1859-1865) está reconvertido en comisaría de policía y estación de autobuses con añadidos y drástica modificación interior. Se observa degradación tanto en la materialidad del edificio como en el uso desvinculado de su uso original ferroviario.

4. Concretamente en la estación Valenciana $^{22}$ se han producido intervenciones tanto mayores como menores profundamente perturbadoras, y muchas de ellas fueron realizadas con posterioridad a la declaración de BIC, siendo la más flagrante la reconversión total del ala derecha del edificio de viajeros. Las intervenciones menores también resultan desgarradoras: sustitución de la marquesina lateral, eliminación de lámparas exteriores originales, cambio del color de fachada, sustitución de carpinterías y vidrios, reparaciones en zócalos pétreos, etc. En definitiva, pocos espacios quedan reconocibles actualmente en la esta- 
ción. En este caso concreto, habiendo estudiado en profundidad el modo de hacer del arquitecto Demetrio Ribes con motivo de mi tesis doctoral, se reconocen soluciones constructivas, pautas de proyecto, etc., en su obra que, podrian haber indicado el modo de intervenir o los materiales que han debido utilizar. En este caso, observando la trayectoria de las intervenciones realizadas y su impacto en el edificio, el estado actual de uso y las posibilidades de este, quizá sería conveniente -dada la coyuntura actual- replantear, discutir, reinterpretar, consensuar el futuro de este edificio dentro de la operación "Parque central-Estación intermodal".

5. Como conclusión respecto de estas tres intervenciones en tres estaciones protegidas se observa que realmente no se han respetado como estaciones ni tampoco todos los valores tipológicos, materiales, de uso, sociales, etc., que motivaron la protección, sino que, básicamente, la protección parece reducirse generalmente a la conservación de la fachada o de aquellos fragmentos del edificio -ni siquiera muchas veces se protegen espacios completos-donde aparece una manifestación artística convencionalmente valorable (lienzo, mosaico, escultura, etc.) Tampoco la protección jurídica garantiza la conservación efectiva ni evita estragos (por ejemplo, en el ala derecha del edificio de viajeros de la estación de Valencia). Otra de las conclusiones es que, en la estación de Valencia -la única de las tres que mantiene el uso ferroviario- a pesar de lo traumático de las intervenciones, mantener el uso ferroviario ha evitado destrozos mayores.

6. En otro nivel de intervención podría situarse el caso de la estación de Gijón, reconvertida en parte del Museo del Ferrocarril de la ciudad $^{23}$ pero a la que se le adosa una ampliación que realza, por contraste, el viejo edificio. Esta queda incorporada al nuevo edificio que alberga el museo pero mantiene su identidad. Sutiles detalles como el tipo de carpintería, el color de la ampliación, los volúmenes, etc., manifiestan respeto hacia el edificio preexistente mantenido con dignidad.

7. Otro grupo podría ser el formado por las estaciones de Canfranc, Leon, Burgos y Zaragoza-Arrabal porque ninguna de ellas mantiene el uso ferroviario, para ellas se pueden establecer secuencias cronológicas respecto a posibles soluciones de intervención: en el caso de Canfranc ${ }^{24}$ su uso futuro está por decidirse; León, recien-

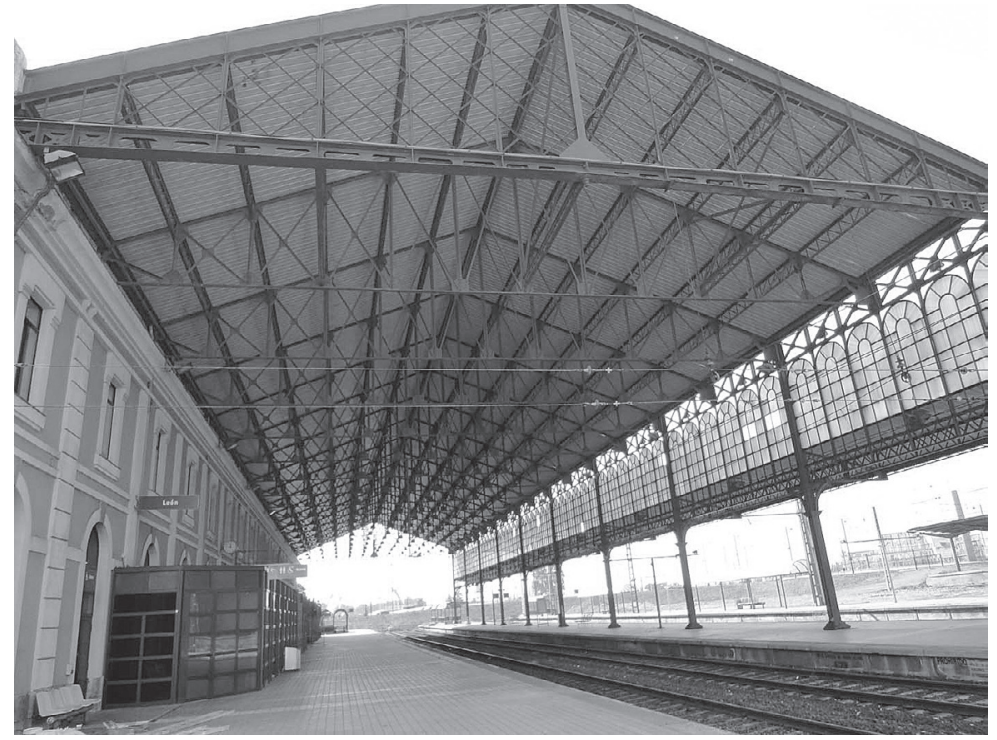

temente ha dejado de funcionar como estación y permanece sin uso. En ella se puede observar, como positivo, su exquisita estructura metálica de la cubierta de andenes y vías, así como esos pequeños detalles modernistas (posiblemente obra de D. Ribes); como negativo, se destacan los disonantes apósitos (con respecto a la volumetría original, espacialidad, materiales, composición, visión conjunta etc.) que constituyen el cuerpo de aluminio anodizado y vidrio añadido en la fachada que recaen en las vías y el acceso principal. Ello da pie a que tampoco se preste atención a intervenciones menores como sustitución de carpinterías, color de fachada, instalaciones vistas, etc., y, en consecuencia, se trasmita una imagen degradante del edificio que, en la teoría, se declara como monumento; Burgos ${ }^{25}$, está en proceso de intervención, pero las vías, parte del entorno y la marquesina han desaparecido: y por último, Zaragoza-Arrabal, con una marquesina descontextualizada y amputada, fue transformada interiormente y convertida en centro cívico donde solo las fachadas y especialmente el letrero que corona el acceso principal recuerdan que se trataba de una estación. Este se constituiría en un ejemplo de reutilización de edificio por el ahorro en la inversión, pero en el que prácticamente ninguno de los valores tangibles e intangibles que justifican lo monumental de las estaciones prevalece.

8. Observar cómo han resultado las distintas soluciones realizadas en su día permite discernir sobre su idoneidad. Soluciones como la de Zaragoza-Arrabal parecen ser el futuro de la estación de Burgos y quizá también de la de León,
Figura 5:

Estación de León.

Fachada interior $y$ cubierta sobre los andenes y vías

Fuente:

Autora, 2010

23 El proyecto del Museo del Ferrocarril fue obra de los arquitectos E. Perea y M. y E. Hernández Sande en 1996, y fue premiado por el Colegio de Arquitectos de Asturias.

24 Fue rehabilitada la cubierta y consolidada la estructura (Arq. J. M. Pérez Latorre). Las obras de conversión en hotel fueron detenidas desde 2009. Actualmente fue vendida a la comunidad de Aragón. El Gobierno de Aragón baraja varios proyectos para la estación, como el Museo-Escuela Internacional del Ferrocarril, la actividad hotelera, una universidad de invierno 0 abrir espacios comerciales y de ocio.

25 Está en proceso de adjudicación y de redacción de proyecto. Fechas previstas construcción: finales 2013 y 2014. Inauguración: 2015. Uso previsto: centro de nuevas tecnologías para uso sobre el estado del edificio fue realizado por el Colegio de Arquitectos de Burgos en 2012. infantil y juvenil. El peritaje 


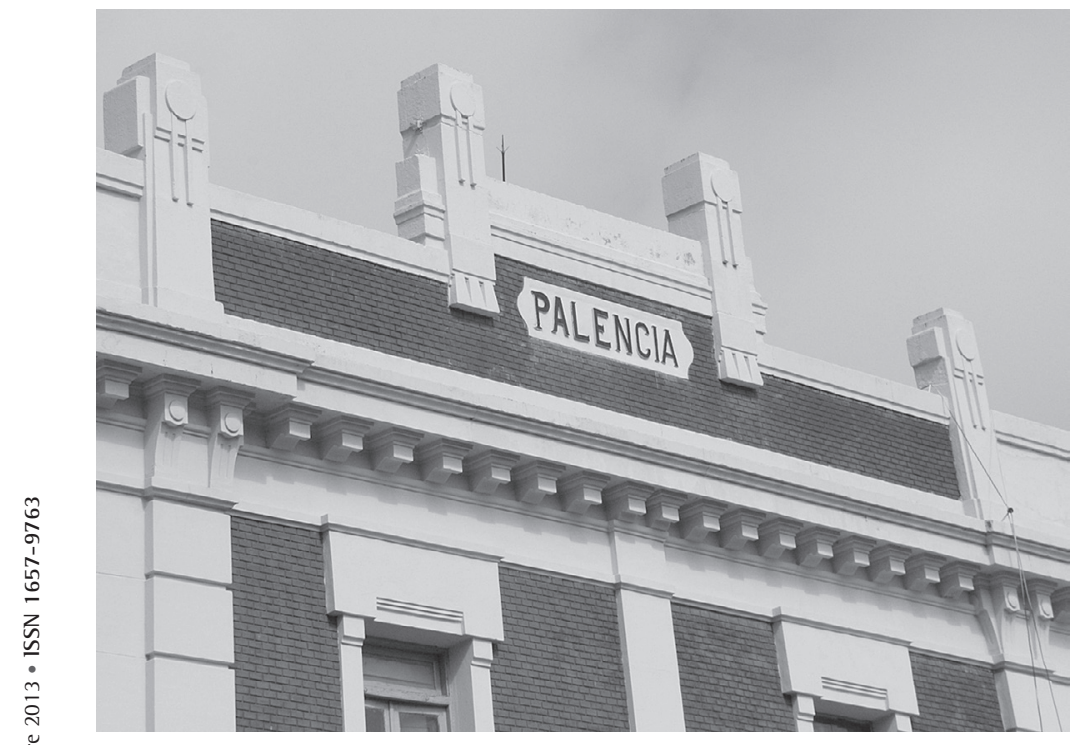

Figura 6:

Estación de Palencia. Detalle de remate en la fachada con el característico ladrillo rojo

Fuente:

Autora, 2011

26 Fue rehabilitada recientemente, específicamente el antiguo edificio de viajeros "estación de $2^{\mathrm{a}}$ clase". Programa: intervenciones en obras públicas y en patrimonio edificado relacionado con ellas con valor patrimonial. Fecha del acuerdo de aprobación comisión mixta: 25 02-2008 (Fuente: Ministerio de Fomento). Se recuperaron su tipología y los materiales originales. Se hizo limpieza y consolidación de la piedra.

27 A pesar de que la toma de conciencia sobre este patrimonio es muy lenta algo se va avanzando. Es destacable en este sentido que estuvieron a punto de derribarse las estaciones de Atocha, Valencia-Nord y

Bilbao-Concordia y ello actualmente resulta impensable.

28 La obra de J. A. Sanz y J. Giner, en 1984, respecto de la arquitectura industrial catalana, constituye un punto de inflexión sobre la forma en que en lo sucesivo iba a considerarse el patrimonio industrial (Calderón, 2007). la inclusión de elementos nuevos, justificados por mejoras y "modernizaciones", tales como marquesinas en los accesos y señalización, con materialidad extraña para el edificio preexistente que degrada profundamente y cambia su imagen. También son frecuentes las sustituciones de materiales de acabado de cubierta, revestimientos y carpinterías, que no buscan ninguna coherencia con lo preexistente. La misma insensibilidad se muestra sobre las más nimias operaciones de mantenimiento. No se justifican estas operaciones por un menor coste pues existe una nula conciencia de que se trata de edificios con un valor inherente, lo que generaliza este tipo de actuaciones tanto en los edificios no protegidos como en aquellos que sí lo están. Finalmente, estas alienaciones aportan, en definitiva, a la conciencia colectiva, la sensación de poco cuidado e interés, de degradación y poca importancia. ¿Sería tolerable un acceso de panel de aluminio en la fachada principal de la catedral de León?

Se destacan entre el grupo dos estaciones: Medina del Campo, sobre la que no se observan elementos distorsionantes en exceso (el más llamativo es la chapa dispuesta en la cubierta de andenes y vías); y Miranda de Ebro, recientemente rehabilitada y sobre la que han pesado criterios de recuperación de tipología y materiales originales. Se tratan pues de un indicio de toma de conciencia sobre cómo actuar en elementos patrimoniales, muy distante al modo en el que se han planteado históricamente los planes de modernización de estaciones $^{27}$.

11. Finalmente, al observar el grupo de estaciones seleccionadas, en su conjunto existe una mayor degradación del edificio en aquellos casos donde ha desaparecido el uso ferroviario o relacionado con el mismo (E. P. Pío o Barcelona Nord), no ocurre lo mismo con el entorno, cuya mejora se produce cuando se eliminan elementos de la infraestructura viaria o se cambia según usos determinados (por ejemplo, se pueden comparar los entornos de la estación de Barcelona Nord y la Zaragoza-Arrabal o, próximamente, la de Burgos). Es decir, parece que persista esa tendencia general de entender lo ferroviario como algo negativo y molesto, sin embargo existen numerosas experiencias europeas de regeneración del entorno con la integración del ferrocarril (Santos y Ganges, 2007).

\section{Discusión}

1. La singularidad arquitectónica del patrimonio industrial empieza a analizarse específicamente en España a partir de los años ochenta ${ }^{28}$, pero respecto de las estaciones de ferrocarril fue la exposición "El mundo de las estaciones" (P. Navascués - 1. Aguilar. Madrid, 1980) la que realizó una primera llamada de atención sobre el tema. Es evidente que desde entonces hasta ahora, se ha avanzado en concienciación y actuaciones sobre las estaciones: desde salvar del derribo de algunas históricas estaciones, hasta intervenciones anónimas que buscan preservar los valores de la obra original. Pero el análisis realizado sobre las 
intervenciones realizadas en las estaciones seleccionadas muestra que todavía no es suficiente el grado de concienciación ni tampoco se ha dado en todos los niveles (sociedad, políticos, técnicos, etc.), ya que, a pesar del buen número de manifestaciones teóricas al respecto, en conjunto se está produciendo una importante degradación paulatina de este patrimonio, incluidos aquellos edificios sobre los que recae el máximo nivel de protección jurídica (es el caso de la estación de Valencia).

2. Respecto del tándem uso-revalorización monumental y su afectación sobre las estaciones, se puede precisar, tras los casos estudiados, que si bien el uso cotidiano no contribuye al incremento de la consideración monumental ${ }^{29}$, el cambio de uso y la desvinculación total del edificio con lo ferroviario produce efectos devastadores. Obsérvese para ello lo acontecido en las estaciones de Gijón y de Madrid-P. Pío o Barcelona-Nord. Rasgos como la amplitud y diafanidad de espacios, la flexibilidad que las estructuras provocan, etc., característicos de las estaciones, facilita la adopción del uso más conveniente según estudios económicos, pero sin tener en cuenta la conveniencia de la preservación del monumento. La intervención barcelonesa permite demostrar la gran variabilidad de uso que admite el espacio bajo las grandes bóvedas de las estaciones debido, únicamente, a las amplias luces existentes, pero ello no se está valorando per se como una innovación tipológica y un avance en las estructuras y uso de los materiales, sino, simplemente, se están utilizando estos espacios posiblemente con criterios de ahorro económico. Tampoco la banalización y exposición superficial de los edificios para consumo turístico resulta efectiva (por ejemplo, Madrid-P. Pío). Por eso, las directrices pedagógicas deben ser la base de estos proyectos turísticos.

3. Resulta fundamental incidir en la gravedad que supone la pérdida total de referencias y contexto. La suma de pequeños y aparentemente insignificantes cambios materiales; los grandes cambios materiales; transformaciones estéticas y espaciales; y la eliminación total del entorno, suponen la pérdida de significado del edificio. También la ruptura con la historia y su razón de ser que permiten la justificación paulatina de la falta de mantenimiento, el abandono, la degradación y la demolición sin mayor repercusión. Por eso resulta fundamental el conocimiento exhaustivo de la documentación existente acerca del edifi- cio que se quiere intervenir, ya que esto permite conocer la obra y vislumbrar posibles soluciones acordes con lo original.

4. Existe una total falta de coordinación entre los distintos organismos públicos, bien sean estatales o autonómicos, de forma que no existe una base de bienes de interés cultural (inmuebles y muebles) única donde, además, queden perfectamente definidas las categorías así como los criterios de búsqueda y palabras clave. Tampoco en muchos casos se han tramitado en su totalidad los expedientes de declaración de bien de interés cultural incoados. Igualmente, las leyes autonómicas tratan de muy diferente modo al patrimonio industrial faltando unicidad en los criterios. Es especialmente difícil conocer un registro exhaustivo del patrimonio industrial, e imposible respecto de las estaciones de ferrocarril ya que de estas no existe ningún inventario específico. Es decir, a pesar de las numerosas declaraciones, de los avances legislativos, al menos en lo que atañe a las estaciones, queda mucho por concretar para poder empezar a protegerlas realmente.

5. Por último, el alcance de la incoación o declaración de BIC aplicado al caso en estudio, evita la demolición de un determinado edificio (la declaración como "monumento" de las estaciones, excluye al conjunto de edificios que la conforman realmente) pero no resulta efectiva para el resto de actuaciones como muestran los casos estudiados.

\section{Conclusiones}

1. Es necesario realizar un inventario interdisciplinar de las estaciones, que sea elaborado con unicidad de criterios sobre qué conservar y cómo intervenir, entendiendo que no se trata de elementos aislados sino que estos forman parte de un conjunto de valores culturales amplios (Benito del Pozo, 2012). Además de esto, es fundamental la elaboración de una especie de "libro del edificio" práctico -realizado a partir de un riguroso y exhaustivo estudio de la documentación existente-, disponible para los técnicos encargados del mantenimiento o de cualquier intervención, donde se detallen los valores de la obra así como sus características tipológicas, compositivas, estéticas y materiales, de modo que se eviten las pequeñas intervenciones disonantes pero altamente destructivas para el edificio y para su concepción social.
29 Nótese que esto ocurre con los usos industriales en general y también con el uso ferroviario en particular, al que se le atribuyen históricamente connotaciones negativas. No ocurre esto con otros usos (por ejemplo, el culto en las catedrales). 
2. Resulta fundamental analizar cuidadosamente el uso futuro no solo desde perspectivas económicas y tenerlo perfectamente definido con anterioridad a la intervención. Tras los casos analizados, se observa que no resulta apropiado para la conservación de las estaciones desvincularlas completamente de los usos ferroviarios o relacionados con estos. Respecto del uso turístico, la simple y trivial exposición no sirve. Criterios educativos deben regir estos usos.

3. Es especialmente importante, en el caso de las estaciones, conservar o intervenir el conjunto. Mantener un elemento de un determinado edificio del conjunto de la estación (por ejemplo, mantener las fachadas del edificio de viajeros de la estación Príncipe Pío) no tiene sentido, ya que se desvincula la intervención con la razón de ser y la esencia del edificio original. Me planteo qué tiene que ver conservar unas fachadas con lo que es una estación y con lo que históricamente ha supuesto el ferrocarril en España.

4. Reutilizar no significa proteger. La tipología de las estaciones permite muy variados usos, aunque algunos de ellos no contribuyen a su protección. Esta es, simplemente, la utilización, con criterios economicistas, de un determinado edificio de la estación como contenedor. El caso de Barcelona-Nord supone un claro ejemplo.

5. Debe prestarse atención a las intervenciones menores (carpinterías, instalaciones, acabados, mobiliario, señalización, etc.) y a aquellas que afectan a elementos meramente funcionales, no valorados o a los que se les atribuyen determinados atributos artísticos, ya que contribuyen a la degradación paulatina y percepción negativa de las estaciones. Estas, en el caso valenciano, resultaron, en conjunto, sumamente perjudiciales tanto desde el punto de vista arquitectónico como sociológico.

6. En otro orden de cosas, debe revisarse con actitud crítica la efectividad real de la protección jurídica que implica la incoación o declaración de BIC. En el caso de las estaciones analizadas, su efectividad práctica se ha ceñido básicamente a evitar la demolición pero no a impedir brutales intervenciones en distinto grado. La búsqueda de una mayor unificación de criterios respecto de la legislación en materia de protección del patrimonio y en la elaboración de los distintos registros resulta fundamental como punto de partida.

7. Por último, como conclusión esencial en conjunto, el estudio analizado -representativo y extrapolable-demuestra que la protección actual de las estaciones no es efectiva. Estaciones tan uniformes tipológicamente como las de Norte se protegen jurídicamente en distintos niveles según las CCAA (Comunidades Autónomas), sin detectarse de forma clara y unívoca los motivos que fundamentan la protección; también se intervienen con criterios distintos -incluso opuestos- y no existe una visión global sobre el conjunto de estas estaciones que facilite posibles vías de actuación. El discurso teórico no llega a las aplicaciones prácticas y concretas. Eso sí, en la intervención de la estación de Canfranc, aún tenemos una gran posibilidad de revertir esta tendencia.

\section{Referencias}

Aguilar 1. (2006). Historia del transporte y de la obra pública como recurso cultural. El museo del transporte y del territorio en la Comunidad Valenciana. Scripta Nova, X(218).

Aguilar, 1. (2012). Lugares e itinerarios de las obras públicas: el paisaje valenciano [catálogo de exposición]. Valencia: Conselleria de Infraestructuras, Territorio y Medio Ambiente.

Alonso, P. y Benito del Pozo, P. (2012). Industrial Heritage and Place ldentity in Spain: from Monuments to Landscapes. Geographical Review, 102, 446-465.

Álvarez, M. A. (2002). Patrimonio industrial: lugares de la memoria. Proyectos de reutilización en industrias culturales. Turismo y museos. Incuna, 87-108.

Sobre esta publicación se encontró:

Álvarez, M. A. (2008). Patrimonio industrial: un futuro para el pasado desde la visión europea. Apuntes, 21(1), 6-25.

Benito del Pozo, P. (2002). Patrimonio industrial y cultural del territorio. Boletín de la Asociación de Geógrafos Españoles, 34, 213-228.

Benito del Pozo, P. (2012). Territorio, paisaje y herencia industrial: debates y acciones en el contexto europeo. Documents d'anàlisi geogràfica, 58(3), 443-457.

Benito del Pozo, P. y López, A. (2008). Patrimonio industrial y nuevas perspectivas funcionales para las ciudades en reestructuración. Estudios Geográficos, LXIX(264).

Bonet, Y. (1992). Recuperación de la estación de la Concordia. Actas del VIIl Congreso Inter- 
nacional para la Conservación del Patrimonio Industrial, 117-121.

Calderón, B. (2007). Nuevos usos para el patrimonio arquitectónico industrial en Valladolid: completar equipamientos y generar valor. Scripta Nova, XI(247).

Calduch, J. (2009). El declive de la arquitectura moderna: deterioro, obsolescencia, ruina. Palapa, IV (II), pp. 29-43.

López, C. (1999). Interrelación de las Categorías Legales de Protección del Patrimonio Cultural en España. Ph. Boletín del Instituto Andaluz del Patrimonio Histórico, 27, 83-90.

López, M. (2006). Las estaciones: práctica y poética. Revista del Ministerio de Fomento, 553, 4-9.

Llull, J. (2005). Evolución del concepto y de la significación social del patrimonio cultural. Arte, individuo y sociedad, 17, 175-204.
Menéndez, F. et al. (1996). Príncipe Pío: renovación para la intermodalidad. Madrid: Fundación de los Ferrocarriles Españoles.

Pardo, C. J. (2010). El patrimonio industrial en España: análisis turístico y significado territorial de algunos proyectos de recuperación. Boletín de la Asociación de Geógrafos Españoles, 53, 239-264.

Riegl, A. (1987). El culto moderno a los monumentos: caracteres y origen. Madrid: Visor.

Santos y Ganges, L. (2007). Urbanismo y ferrocarril. La construcción del espacio ferroviario en las ciudades medias españolas. Madrid: Fundación de los Ferrocarriles Españoles.

Siza, Á. (2011). Criterios de intervención en el patrimonio arquitectónico del siglo XX [conferencia]. Conferencia Internacional sobre criterios de intervención en el patrimonio arquitectónico del siglo XX (CAH2OthC). Documento de Madrid, 183-189.

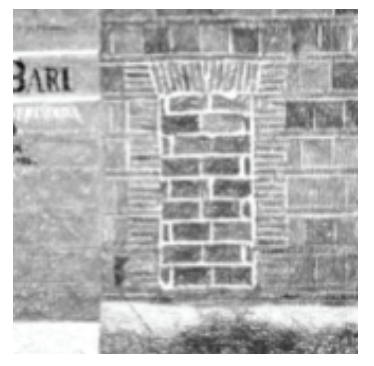

